\title{
Performance Evaluation of Integrated Access and Backhaul in 5G Networks
}

\author{
Philippe Fabian $^{1}$, Georgios Z. Papadopoulos ${ }^{1,2}$, Patrick Savelli ${ }^{1}$, Bernard Cousin ${ }^{3}$ \\ ${ }^{1}$ Institute of Research and Technology $\mathrm{b}<>$ com, Advanced Connectivity Lab, Rennes, France \\ ${ }^{2}$ IMT Atlantique, IRISA, Rennes, France \\ ${ }^{3}$ University of Rennes 1, IRISA, Rennes, France
}

\begin{abstract}
Integrated Access and Backhaul (IAB) allows to ease the deployment of gNodeBs (gNBs) in a $5 \mathrm{G}$ network by connecting them using cellular connectivity. The radio frequencies to achieve such a result can be the same as the ones used to connect User Equipment (UE) (in-band) or specific ones can be reserved (out-of-band). Both sub $6 \mathrm{GHz}$ and millimeter wave (mmWave) frequencies can be used. IAB is relatively new and several contributions have been studying the lower layers (i.e. physical and link) in order to improve performance in scheduling and resource allocation. However, performance results are also very dependent on how the routing is implemented at the network layer which is an aspect that is not much studied in the state of the art. In this paper, we evaluate the performance of several IAB topologies against a topology without IAB-nodes. Results confirm the conclusions of the state of the art by showing that adding IABnodes significantly improve performance in terms of delay (more than $90 \mathrm{~ms}$ shorter delay) and Packet Delivery Ratio (PDR) (by up to $15 \%$ ), and also allows to extend the coverage area. These results reveal the high potential of IAB that could be improved even more using efficient routing.

Index Terms-Integrated Access and Backhaul, 5G, 3GPP, Backhaul Adaptation Protocol, IAB, Routing.
\end{abstract}

\section{INTRODUCTION}

$5 \mathrm{G}$ networks are already being deployed as of the writing of this paper. Compared to $4 \mathrm{G}, 5 \mathrm{G}$ provides better performance overall [1]. This, in turn, allows to extend current scenarios (higher density of devices, better support for mobility, higher throughput, etc.) and to enable new services such as Industry 4.0. The shorter range due to higher frequencies in $5 \mathrm{G}$ forces Internet Service Providers (ISPs) to deploy more antennae/gNBs in a given location (higher density). Doing so could imply the construction of costly wired links to connect the gNBs [2], [3]. However, thanks to IAB, it is possible to avoid this cost by connecting antennae with cellular connectivity [1].

IAB uses the Backhaul Adaptation Protocol (BAP) [4] to route packets through the IAB topology (typically a tree, see Sec. II). The root is called Donor [5] and is a gNB connected to the Core Network $(\mathrm{CN})$ through a wired link. It manages the topology by assigning BAP addresses to IAB-nodes ( $\mathrm{gNBs}$ connect wirelessly to the Donor through one or several hops) and by setting paths on which packets are sent. It is possible to have several paths for a source-destination tuple. This increases reliability (for instance, in the case of a failure or radio interference) and flexibility (e.g. to dynamically change paths according to user needs).

The Donor is composed of a Central Unit-Control Plane (CU-CP), a CU-User Plane (CU-UP), one or several Distributed Units (DUs) and other functions depending on current needs

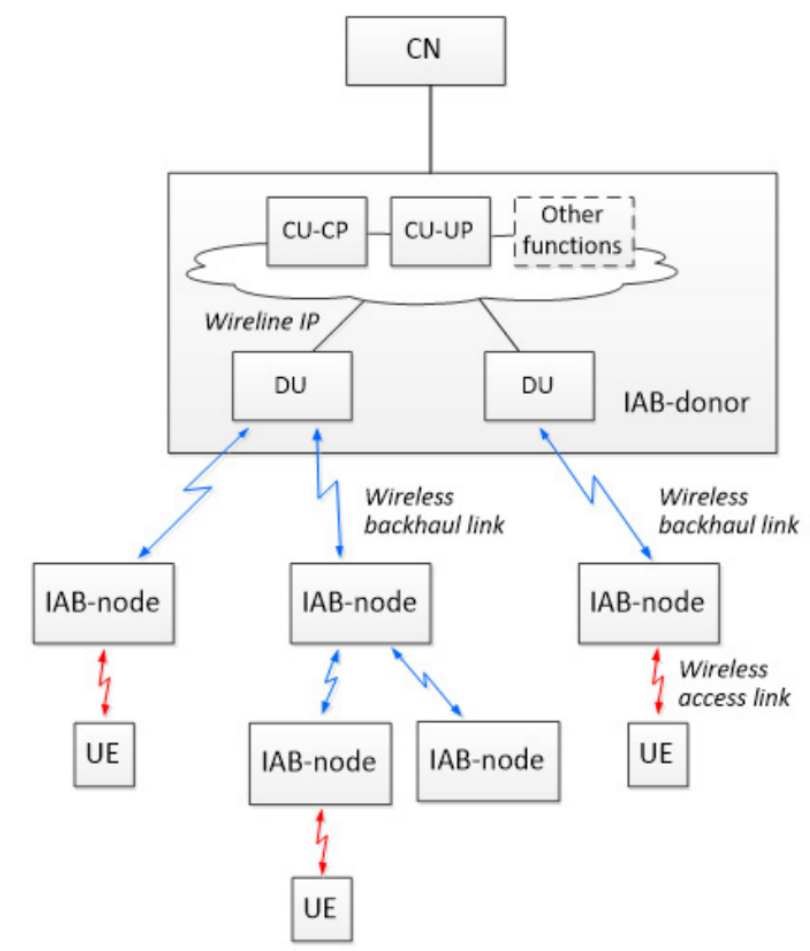

Fig. 1: An example of an IAB topology. Blue links represent backhaul connections whereas red links represent access connections. This figure is taken from [1].

(see Fig. 1 for an example of an IAB-Donor). Note that all these components are not necessarily on the same physical node, thanks to the extensive use of virtualization in 5G. This is useful to ensure better reliability (in case of equipment failure), to start/stop functions according to needs or to use additional physical resources whenever the demand increases and current hardware can not process requests fast enough. The DU part allows children nodes to connect to the current node. A child can either be an IAB-node or a UE. Each IAB-node has two parts: DU and Mobile Termination (MT). The DU part serves the same function as the one on the Donor (providing connectivity to IAB-nodes and UEs). The MT part is used to connect to the parent node (see [1] for more details). The IAB topology and consequently the BAP protocol are transparent from the point of view of UEs.

Fig. 1 displays an example of IAB topology with 1 Donor, 5 IAB-nodes and 3 UEs. The DU and MT parts of the IAB-nodes are not shown on this figure, though. As mentioned earlier, the 
default topology is tree-shaped which makes upstream routing simpler because traffic only needs to be sent to the parent at each hop. However, routing is still needed to forward traffic downards and in the case several paths are created in the upstream direction. The choice of path to use is up to the network operator and thus depends on the chosen algorithms and the local needs.

In this paper, we evaluate the performance of IAB and stress the need to develop new routing solutions. We start by explaining how IAB works at the MAC and BAP layers before discussing state of the art papers. We then evaluate the performance of IAB through simulation and we point out the benefits of it. We also highlight new relevant features of 3GPP release 17 . Our analysis leads to the conclusion that the routing aspect in IAB has a high potential. Indeed, we believe more research and new routing algorithms are needed in order to meet the stringent requirements of very dense $5 \mathrm{G}$ networks with a large number of IAB-nodes.

\section{IAB DETAILS}

In this section, we explain how IAB works. It is worth noting that the MAC layer and BAP are affecting one another. Indeed, the routing decisions taken at the BAP layer might result in sending more (or less) traffic to specific nodes. The resource allocation scheme must be properly set up to avoid congestion. On the other hand, feedback from the MAC to the BAP layer could impact the choice of paths (see Sec. IV-C).

\section{A. Link Layer}

The scheduling scheme needs to take into account interference [1]. We can identify four cases concerning this issue:

- Out-of-band relaying [6]. IAB-nodes can use sub $6 \mathrm{GHz}$ frequencies (more range, more resistant to blockage) to communicate amongst themselves and higher frequencies (mmWave) can be used for transmission between an IAB-node and its attached UEs. Note that this out-ofband separation does not solve the problem but rather decomposes it into two smaller (simpler) problems.

- Time Division Multiplexing (TDM). A node can transmit on the whole range of frequencies but only during a limited time frame. In that case, it is possible to optimize downwards traffic by allocating the first time frames to the parent, then the next ones to the child so it can directly forward data from parent to next child. It is the same, respectively, for upwards traffic (first child, then parent).

- Frequency Division Multiplexing (FDM). In that scheme, a node can transmit at any time but with a restrained bandwidth. As of the writing of this document, TDM is more in use compared to FDM (see Sec. III).

- Spatial Division Multiplexing (SDM). This is possible by using beamforming techniques and allows different nodes to transmit and receive at the same time and on the same frequency. However, if many nodes transmit there will be more interference with increasing transmissions. Also, if we have to consider mobility (especially) on IABnodes, the variation in the distance between nodes might substantially increase the complexity of the beamforming algorithm.
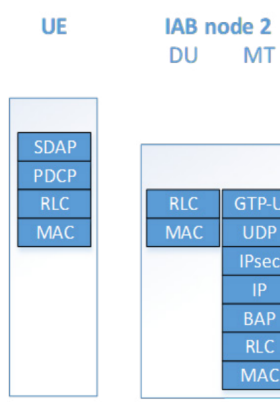

MT
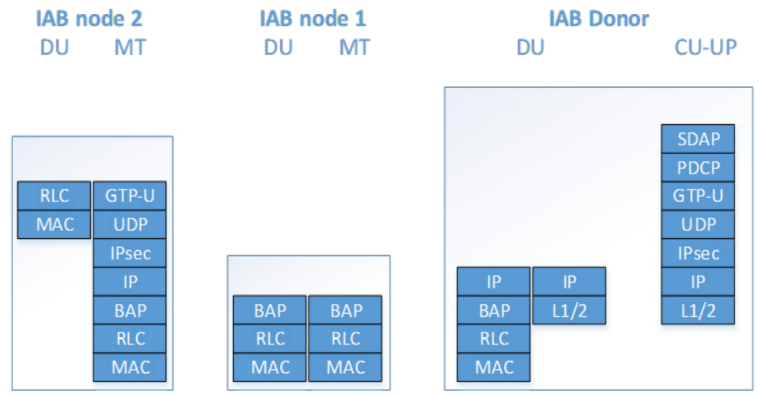

Fig. 2: The BAP layer is located above the RLC layer. This figure is taken from [1] and represents the user plane.

\section{B. BAP Description}

Routing in IAB is done at the BAP layer. Given this contribution focuses on the routing aspect, it is important to properly explain BAP and how it can be used to improve performance. Fig. 2 shows where the BAP layer is located in the network stack. This layer acts in a similar way as Internet Protocol (IP) and IAB-nodes are similar to routers in that they route and forward traffic (to other nodes) upwards or downwards if the packet has not reached its final destination yet. A UE does not have a BAP layer. In addition to routing packets, BAP supports flow control. Other functions are defined, see [4] Sec. 4.4 for more details. The possibility to choose the path to send packets is interesting as it allows to find the best one according to current needs (this will be covered more in details in Sec. IV). A reliable and/or stable default path can be established so there is always at least one possible path to reach the Donor and other nodes.

Fig. 3 illustrates a BAP control Packet Data Unit (PDU) used for flow control feedback. The Routing ID is the DESTINA$T I O N+P A T H$ (from Fig. 4). This packet passes information to the Donor about the buffer usage of a given tuple [destination; path]. The Donor can use this information to adapt the resource allocation scheme if an IAB-node is congested or to avoid sending too much traffic their way. Note that there also exists a flow control PDU that associates an RLC channel ID to a buffer size but it is not shown here. It serves a similar purpose as the flow control PDU depicted on Fig. 3.

Fig. 4 shows a detailed view of a BAP data PDU that is 3 octets is size (plus the size of the data). The two fields of interest to us are DESTINATION (BAP address) and PATH. Both are coded on 10 bits, hence there are 1024 possible paths and there can be up to 1024 IAB-nodes including the Donor.

\section{RELATED WORK}

The following analyses several works aiming at improving some aspects within an IAB topology. However, they mostly focus on the PHY/MAC layers.

Islam et al. [7] study joint resource allocation and relay selection in IAB in order to show that it improves the mean data rate of UEs. They formulate the problem as an optimization one. The objective is to maximize the geometric mean of UEs' data rate. Two constraints are also formulated: the flow conservation (inbound traffic should be equal to outbound traffic) and the resource allocation constraint (a timeslot can not 


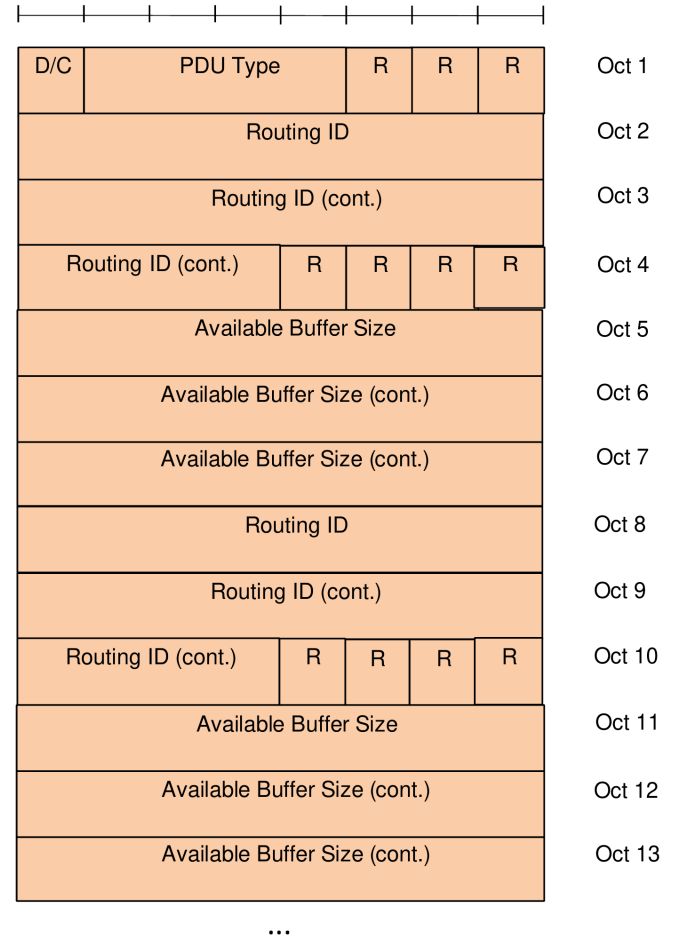

Fig. 3: A BAP control PDU for flow control purposes. This figure is taken from [4]. Note that there are several Routing IDs (octets 2-3 and octets 8-9) with their associated Buffer size (octets 5 to 7 and octets 11 to 13 ).

\begin{tabular}{|c|c|c|c|c|c|}
\hline $\mathrm{D} / \mathrm{C}$ & $\mathrm{R}$ & $R$ & $\mathrm{R}$ & \multicolumn{2}{|c|}{ DESTINATION } \\
\hline \multicolumn{5}{|c|}{ DESTINATION (cont.) } & PATH \\
\hline \multicolumn{6}{|c|}{ PATH (cont.) } \\
\hline \multicolumn{6}{|c|}{ Data } \\
\hline
\end{tabular}

Oct 1

Oct 2

Oct 3

Oct 4

Fig. 4: A BAP data PDU. The "data" field corresponds to the packet from the upper layer (e.g. IP). This figure is taken from [4].

be used simultaneously by two nearby nodes). The approach is based on TDM but authors claim it can be adapted to FDM as well. It is supposed that directional antennae are used. The authors compare a spanning tree versus mesh topology and conclude mesh yields better performance in terms of UE rate and latency. However, their approach is somewhat theoretical and real aspects such as buffering and mobility are not considered. Also, the connection of UEs is done through signal strength only.

In [8], authors formulate an optimization problem taking into account scheduling, resource allocation and routing. They use binary interference classification with the aforementioned elements. They also generate a conflict graph in which wireless links are represented as vertices. Two links are thus in conflict (interference, half-duplex constraint) if connected by an edge.
In practice, slots are allocated to groups of wireless links. Links within a group are not in conflict. Hence, different groups can transmit at the same time (SDM). Then, TDM is used to allocate timeslots to links within each group, proportional to their needs. Power allocation is finally computed to emit at optimal power levels. No interference should occur as they were eliminated thanks to the grouping from the previous steps. The authors also compute the best paths based on the maximum data rate (a high data rate on a link increases its weight, making it more interesting). However, performance evaluation is done using full buffer assumption and no UE mobility is considered.

Authors in [9] design a genetic algorithm to resolve the placement of IAB-nodes and the distribution of non-IAB dedicated connections. The considered metric is the probability a UE receives satisfying data rate according to its needs ("coverage probability"). They use a radio model that accounts for path loss and blocking due to objects. This model includes blocking from vegetation as well. There are two genetic algorithms: the first iterates on the set of possible small base stations (i.e. IAB-nodes) to find where to place non-IAB links, and the second considers the possible location sets for the choice of small base stations. It is assumed IAB-nodes do not interfere with one another (directional antennae). Simulations are run to study the convergence of the algorithms and how well they improve performance of the network. However, they assume there is no interference between UEs which can be an issue in a high density setting such as music festivals or sport competitions. The proposed genetic algorithm is compared to exhaustive search only in terms of complexity. The proposed solution is based on planning the deployment of IAB-nodes: in a more realistic context it might not be possible to choose the locations of base stations, or some exceptional situations could degrade the system, making the planning obsolete or not usable. Also, although the theoretical model considers every UE has a potential need in terms of data rate that is different, this is not considered in the simulations, where the highest achievable data rates are studied. This is not adapted in the case where a differentiation of service is needed.

Pagin et al. [10] design a semi-centralized scheduling algorithm where the Donor decides how the scheduling will be achieved. Then, each IAB-node is free to decide on how the actual MAC level scheduling is done locally. That is, some resources are allocated to a node and it decides how to use them to serve its UEs and children IAB-nodes. The allocation from the Donor is done through a priority scheme where nodes receive resource allocation in order of their position in the priority list (a node will get resources if there are some left). However, given the goal of this contribution, the authors have abstractly merged all UEs attached to one IAB-node into one node connected through one edge. Hence, individual UEs are not modeled.

In [11], the author proposes to improve routing in IAB networks through two mechanisms. The first one is to use handover features from $5 \mathrm{G}$ specifications to change the parent of IAB-nodes in order to load balance the IAB topology. The second mechanism is the modification of the length of the transmission phases: an IAB-node can be allowed to transmit (upstream/downstream) more or less depending on the 


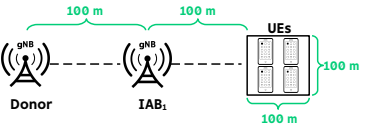

(a) Scenario 1: there is 1 IAB-node between the Donor and UEs. Results are shown on Figs. 6a and $6 b$.

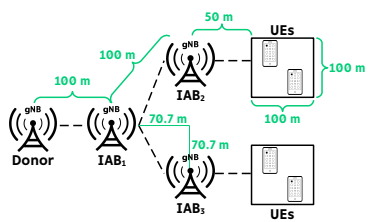

(c) Scenario 3: 3 IABnodes placed in a Y-shape topology. Results are depicted on Figs. $7 a$ and $7 b$.

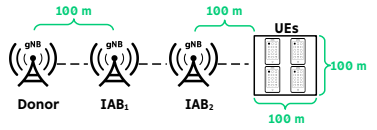

(b) In scenario 2, there are 2 IAB-nodes between the Donor and UEs. Results are shown on Figs. 6c and $6 d$.

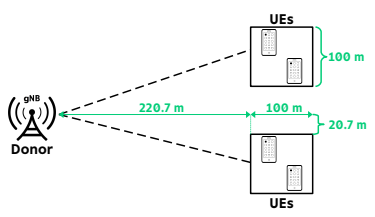

(d) Scenario 4: There are no IAB-node. Results are shown on Figs. 7c and 7d.
Fig. 5: The four studied scenarios.

traffic. This allows to better utilize the time slots by assigning them where there is a higher need. However, only the data rate of UEs is considered to select links in the performance evaluation. This can be a problem when differentiation of service is required. No UE mobility is considered and the phase modification is run once after a handover happened. That is, if handovers are not possible for a relatively long duration, the load balancing can be compromised.

Polese et al. [12] consider the problem of selecting the best path from any IAB-node to a node connected (wired) to the core network. They define several strategies for an IAB-node to select a parent: highest SNR, lowest number of hops to reach a wired node, lowest number of hops (node with highest SNR closer to wired node) and highest possible data rate. However, considering the data rate and hop count is not enough to ensure Quality of Service (QoS) in realistic cases, and the results only show a study on the number of hops from IAB-nodes to a wired node.

As mentioned at the beginning of this section, these works focus on the lower layers (especially the MAC layer). Routing is not thoroughly studied and neither are routing algorithms. The next section will discuss these.

\section{Study of IAB and Possible Developments}

\section{A. On the Validity of IAB}

This subsection shows the results of four different scenarios. In scenarios 1 and 2 the Donor, IAB-node(s) and UEs are placed on a line. There is 1 IAB-node in scenario 1 (Fig. 5a) and there are 2 IAB-nodes in scenario 2 (Fig. 5b). All UEs are randomly placed inside a $100 \mathrm{~m}$ by $100 \mathrm{~m}$ "box". The distance between the Donor, each IAB-node(s) and the box of UEs is $100 \mathrm{~m}$. In scenario 3 (Fig. 5c), UEs are split in two groups (each with half the total number of UEs). The distance between the Donor and IAB-nodes is $100 \mathrm{~m}$. Thus, $\mathrm{IAB}_{2}$ and $\mathrm{IAB}_{3}$ have a horizontal and vertical distance of about $70.7 \mathrm{~m}$
TABLE I: Values of parameters. The physical and MAC models are described in [13]. ${ }^{\dagger}$ Only in scenario $3 .{ }^{\star}$ Only relevant in scenario 4.

\begin{tabular}{|c|c|c|}
\hline Parameter Name & Value (scenarios 1 \& 2) & Value (scenarios 3 \& 4) \\
\hline Number of IABs & 1 or 2 & 0 or 3 \\
Number of UEs & {$[2 ; 8]$ step 2 } & {$[2 ; 8]$ step 2} \\
Traffic load (packet/s) & {$\left[10^{4} ; 10^{5}\right]$ step 2500} & {$\left[10^{4} ; 10^{5}\right]$ step 2500} \\
Packet size & 1500 bytes & 1500 bytes \\
Traffic model & CBA & CBA \\
Distance between IABs & $100 \mathrm{~m}$ & $100 \mathrm{~m}$ \\
Dist. UEs \& nearest IAB & {$[100 ; 210.80] \mathrm{m}$} & {$[50 ; 160.80] \mathrm{m}^{\dagger}$} \\
Dist. UEs \& Donor & - & {$[221.67 ; 342.66] \mathrm{m}$} \\
Height of IABs & $10 \mathrm{~m}$ & $10 \mathrm{~m}$ \\
Height of UEs & $1.5 \mathrm{~m}$ & $1.5 \mathrm{~m}$ \\
Simulation time & $3.6 \mathrm{~s}$ & $3.6 \mathrm{~s}$ \\
Number of repetitions/runs & 10 & 10 \\
\hline
\end{tabular}

from $\mathrm{IAB}_{1}$. The distance between $\mathrm{IAB}_{2}$ and $\mathrm{IAB}_{3}$ and their respective "box" of UEs is $50 \mathrm{~m}$. In scenario 4 (Fig. $5 \mathrm{~d}$ ), the placement of the Donor and the boxes of UEs is the same as in scenario 3 but the three IAB-nodes have been removed. In all scenarios, distances are shown on Fig. 5. UEs are connected to the closest IAB-node (or the Donor in scenario 4). Simulations are done using the ns-3 simulator with the mmWave [13] (5G) and IAB [14] extensions. Table I depicts the most relevant parameters of the simulations.

The $x$-axis on all graphs (Figs. 6 and 7) represent the traffic load (bold text in Table I). Packets are sent at a Constant Bit Rate (CBA). A UE has a minimum distance from an IABnode (Donor in scenario 4) when it is on the center-left side of the box (or, in scenario 4, the lower-left corner for the upper box and upper-left corner for the lower box). The maximum distance is when a UE is on one of the right (lower or upper) corners of the box (except for scenario 4, where maximum distance is reached if a UE is placed on the upper-right corner for the upper box or the lower-right corner for the lower box). In scenario 4 the boxes of UEs are closer by $50 \mathrm{~m}$ to the left because the loss rate is greater (c.f. Figs. 7c and 7d). Scenario 3 also has a $50 \mathrm{~m}$ shorter distance (compared to scenarios 1 and 2) to mirror scenario 4. UEs run a UDP client connected to a server on Internet. The delay and Packet Delivery Ratio (PDR) shown on the following graphs are computed from data packets in upload (UEs to server). The connection between the server and the Donor is a link at 10 Gbps with a delay of 2 ms.

The delay is computed from packets reaching the destination: if few packets arrive the results are less significant. In the graphs below, the value of one data point is the average of all runs. The value of one run is the averaged value (for delay) of all packets generated during that run. There is one PDR value per run. Concerning the asymmetrical error bars, the standard deviation is used. For each data point, the values of runs above (resp. below) the average have their standard deviation computed. The value of this standard deviation is the length of the error bar above (resp. below) the data point. Asymmetrical bars are more precise than symmetrical ones and prevent unrealistic values (e.g. negative delays).

Figs. 6a and $6 \mathrm{~b}$ depict the case with 1 IAB-node. The PDR (Fig. 6a) is stable at $100 \%$ until the total traffic load reaches about $1.5 \mathrm{Gbps}$ ( 2 UEs times $7.5 \cdot 10^{8} \mathrm{bit} / \mathrm{s}$, or 4 UEs times 

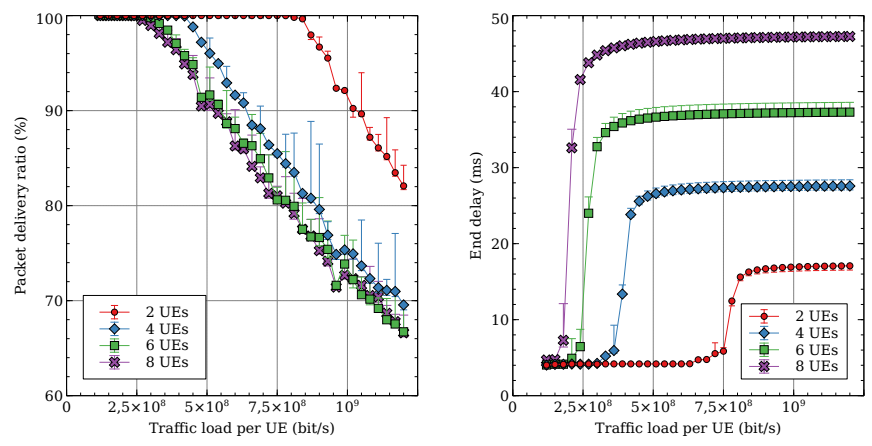

(a) The PDR for scenario 1 (see Fig. 5a).

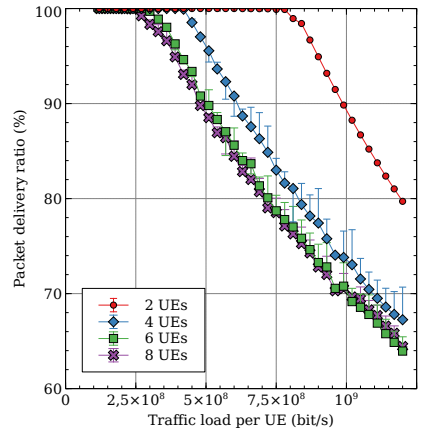

(c) The PDR scenario 2 (see Fig. 5b). 1 (see Fig. 5a).

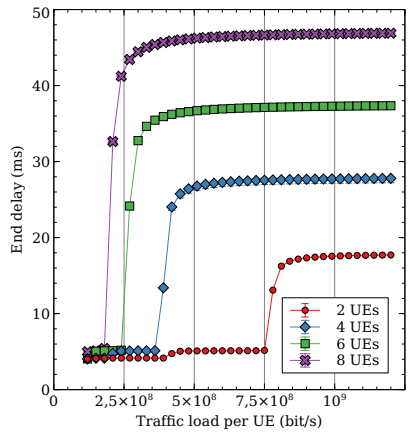

(d) The delay scenario 2 (see Fig. 5b).

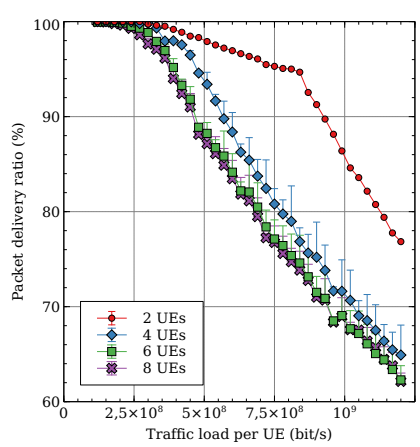

(a) The PDR for scenario 3

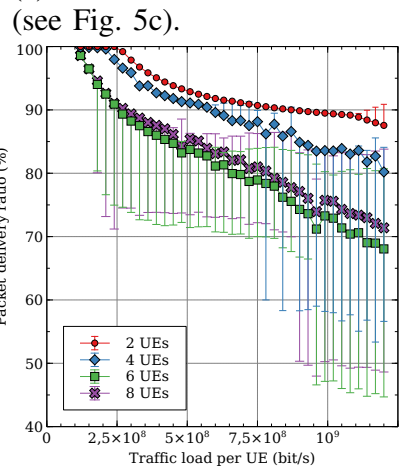

(c) The PDR scenario 4 (see Fig. 5d).

Fig. 6: Values of PDR and delay versus traffic load for scenarios 1 and 2. Note the vertical axis cut on PDR graphs (Figs. 6a and $6 \mathrm{c})$.

$3.75 \cdot 10^{8} \mathrm{bit} / \mathrm{s}$, etc.). The PDR starts to decrease because of congestion and more interference. The maximum achievable throughput is about $1.5 \mathrm{Gbps}$ (this concurs with results from [13]) so the limit is not on the Donor to Internet side (link at 10 Gbps as aforementioned) but rather on the IAB topology. On Fig. 6b, the delay is stable near 4-5 ms until congestion. Then, the value of delay increases to about $17 \mathrm{~ms}$ (2 UEs), $27 \mathrm{~ms}$ (4 UEs), $37 \mathrm{~ms}$ (6 UEs) and $47 \mathrm{~ms}$ (8 UEs). The average delay is higher with more UEs compared to fewer UEs because each one needs to wait for other UEs to be served before its own packets are processed. The delay reaches a top value because buffers are full and no new packets can be sent on the topology (thus the maximum waiting time is reached).

Concerning scenario 2 (Figs. 6c and 6d), the curves are similar to the case with 1 IAB-node (Figs. 6a and 6b). There is a slight difference in the PDR, though, because there is one more hop to reach the Donor and thus the probability for a transmission error is higher. Delays are not significantly impacted because they increase mostly due to congestion.

Results from scenario 3 and 4 are shown on Fig. 7. Figs. $7 \mathrm{a}$ and $7 \mathrm{~b}$ depict the results from scenario 3. They are better compared to scenario 4 (Figs. 7c and 7d). However, they are worse compared to scenarios 1 (Fig. 5a) and 2 (Fig. 5b) because $\mathrm{IAB}_{- \text {node }_{1}}$ receives traffic from two IAB-nodes. Indeed, the PDR on Fig. 7a starts to decrease at about the same values compared to other graphs, but with a steeper slope. The delay with 2 UEs (on Fig. 7b) goes from about 4-6 ms at low charge

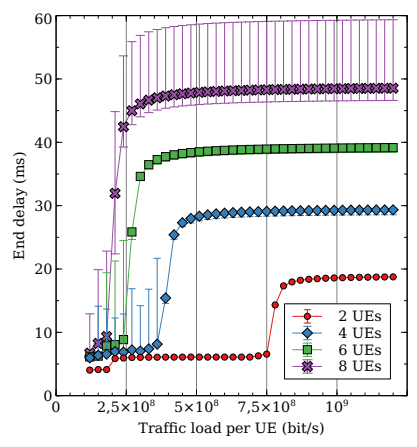

(b) The delay for scenario 3 (see Fig. 5c).

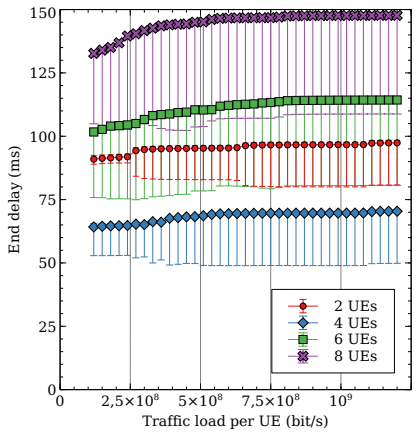

(d) The delay scenario 4 (see Fig. 5d).

Fig. 7: Delay and PDR versus traffic load for scenarios 3 and 4. Note the cut on the vertical axis on PDR graphs (at $60 \%$ for Fig. 7a) and $40 \%$ for Fig. 7c.

up to about $18.75 \mathrm{~ms}$. In comparison, 2 UEs on scenario 1 (Fig. 6b) have an average delay of about 4-5 ms at low charge and the delay increase up to $17.08 \mathrm{~ms}$ at maximum charge.

Scenario 4 has much higher delays (Fig. 7d) compared to the other scenarios (up to $100 \mathrm{~ms}$ more compared to scenario 1, Fig. 6b). The PDR (Fig. 7c), considering error bars, is lower than all other cases (for 6 UEs, about $68.05 \%$ on average and about $44.69 \%$ considering variance). The PDR seems very high (above $85 \%$ at maximum load), but this is due to very few packets able to reach the Donor. Indeed, most are lost and do not even "leave" the $\mathrm{UE}^{1}$. In scenario 4, error bars are longer below the average. This is caused by few packets being correctly transmitted the first time and having a relatively low PDR (Fig. 7c) or delay (Fig. 7d). However, most packets have a value close to the average.

These results highlight the benefits of deploying IAB-nodes for better performance and coverage rather than having long range (lossy) transmissions. More hops slightly decrease PDR (Fig. 6a versus 6c) but delay is not affected as long as there is no congestion. It is also interesting to see that there is a bottleneck when several IAB-nodes send traffic to a common parent: this shows the importance of robust routing on complex topologies with many IAB-nodes to help manage such issues.

\footnotetext{
${ }^{1}$ More precisely, simulations on the line topology with 1 IAB-node gives about 75000 packets at maximum charge whereas the no IAB-node topology yields about 100 packets for the whole run.
} 


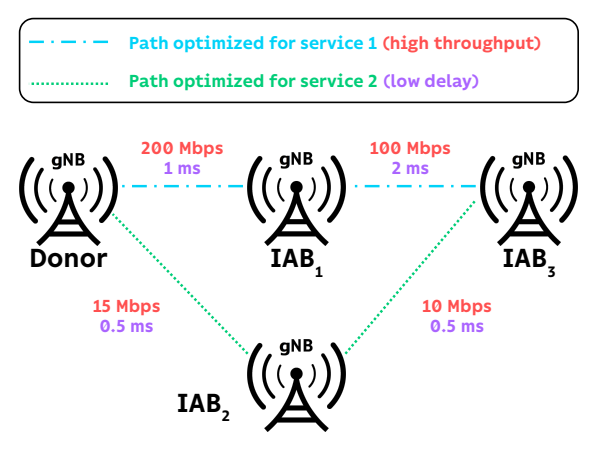

Fig. 8: An example of a topology with 2 paths set up. Both paths can be optimized for a different service, improving QoS.

\section{B. 3GPP Release 17}

Release 17 comes with several new features concerning IAB [15], [16], [17]. These features are expected to positively impact IAB by increasing performance and providing more flexibility and reliability to send data. The most relevant features are summarizedin the following list along with an explanation/example of how each one can be exploited:

- The radio spectrum is expanded to $71 \mathrm{GHz}$ (from 52.6 $\mathrm{GHz}$ ). This offers a higher flexibility in the choice of frequencies and it will increase achievable throughput.

- Better support for simultaneous transmission and reception (i.e. full-duplex). This feature reduces latency and increases spectral efficiency.

- Inter-Donor migration of IAB-nodes. This will allow to dynamically adapt the topology with a higher degree of flexibility compared to intra-Donor migration only.

- Support for non-terrestrial networks. This feature enables the possibility to use drones as IAB-nodes. Disaster recovery or ensuring minimal service during a temporary failure are examples of use cases.

\section{Future Research Axes}

From all of the above, we can deduce a few potential research axes. Two of them are enhanced load-balancing and improved differentiation of service.

In Sec. III, we discussed paper [10] in which the authors propose a solution where the Donor allocates resources to IABnodes. Ultimately, it is up to these nodes to decide how to schedule resources locally. This "micro" optimization could be combined with a more "macro" (global) optimization at the network (routing) level. A routing algorithm might be useful in load-balancing the traffic load given the scheduling scheme.

Another possibility is to use the differentiation of service to establish different (routing) paths. Fig. 8 depicts such an example where two paths have been established. On this example, we suppose service 1 needs a high throughput (e.g. downloading/uploading) and service 2 requires a short delay (such as a Voice over IP (VoIP) application). The blue path (Donor $\leftrightarrow I A B_{1} \leftrightarrow I A B_{3}$ ) can achieve an end-to-end throughput of $100 \mathrm{Mbps}$ and the total delay is $3 \mathrm{~ms}$. On the green path (Donor $\leftrightarrow I A B_{2} \leftrightarrow I A B_{3}$ ), the throughput can go up to $10 \mathrm{Mbps}$ only but the end-to-end delay is $1 \mathrm{~ms}$. The blue path is thus better suited for service 1 and the green path is more adapted for service 2 . Such a solution could help in improving QoS and load-balancing might result as a consequence of choosing different paths to send traffic from the same source-destination tuple (one device can run several applications simultaneously).

\section{CONCLUSIONS}

IAB is a solution with a very high potential. Several studies have been conducted to show how it can be used to improve performance, mainly at the MAC layer. Our results concur with these previous findings by showing improvements in terms of delay and PDR. IAB can also be used to extend coverage area and to dynamically adapt the topology of the access network. 3GPP release 17 will come with several new aspects to enhance the capabilities of IAB and we believe it can be further improved using an efficient routing algorithm.

\section{REFERENCES}

[1] "Innovations in 5G Backhaul Technologies: IAB, HFC \& Fiber," 5G Americas, Tech. Rep., June 2020.

[2] 3GPP, "TR 38.874 v16.0.0, Study on Integrated Access and Backhaul (Release 16)," December 2018.

[3] O. Teyeb, A. Muhammad, G. Mildh, E. Dahlman, F. Barac, and B. Makki, "Integrated access backhauled networks," in 2019 IEEE 90th Vehicular Technology Conference (VTC2019-Fall). IEEE, 2019, pp. 1-5.

[4] 3GPP, "TS 38.340 v16.5.0, Backhaul Adaptation Protocol (BAP) specification (Release 16)," June 2021.

[5] — - "TS 38.300 v16.7.0, NR; NR and NG-RAN Overall Description; Stage 2 (Release 16)," September 2021.

[6] — , "TS 38.174 v16.4.0, Integrated access and backhaul radio transmission and reception (Release 16)," September 2021.

[7] M. N. Islam, N. Abedini, G. Hampel, S. Subramanian, and J. Li, "Investigation of performance in integrated access and backhaul networks," in IEEE INFOCOM 2018-IEEE Conference on Computer Communications Workshops (INFOCOM WKSHPS). IEEE, 2018, pp. 597-602.

[8] Y. Li, J. Luo, R. A. Stirling-Gallacher, and G. Caire, "Integrated access and backhaul optimization for millimeter wave heterogeneous networks," arXiv preprint arXiv:1901.04959, 2019.

[9] C. Madapatha, B. Makki, A. Muhammad, E. Dahlman, M.-S. Alouini, and T. Svensson, "On topology optimization and routing in integrated access and backhaul networks: A genetic algorithm-based approach," arXiv preprint arXiv:2102.07252, 2021.

[10] M. Pagin, T. Zugno, M. Polese, and M. Zorzi, "Resource Management for 5G NR Integrated Access and Backhaul: a Semi-centralized Approach," arXiv preprint arXiv:2102.09938, 2021.

[11] Y.-C. Liang, "Dynamic routing and load balancing techniques for integrated access and backhaul network," Master's thesis, KTH Royal Institute of Technology, 2020.

[12] M. Polese, M. Giordani, A. Roy, D. Castor, and M. Zorzi, "Distributed path selection strategies for integrated access and backhaul at mmwaves," in 2018 IEEE Global Communications Conference (GLOBECOM). IEEE, 2018, pp. 1-7.

[13] M. Mezzavilla, M. Zhang, M. Polese, R. Ford, S. Dutta, S. Rangan, and M. Zorzi, "End-to-end simulation of 5g mmwave networks," IEEE Communications Surveys Tutorials, vol. 20, no. 3, pp. 2237-2263, 2018.

[14] M. Polese, M. Giordani, A. Roy, S. Goyal, D. Castor, and M. Zorzi, "End-to-end simulation of integrated access and backhaul at mmwaves," in 2018 IEEE 23rd International Workshop on Computer Aided Modeling and Design of Communication Links and Networks (CAMAD). IEEE, 2018, pp. 1-7.

[15] E. Dahlman, G. Mildh, S. Parkvall, P. Persson, G. Wikström, and H. Murai, "5G evolution and beyond," IEICE Transactions on Communications, vol. E104.B, pp. 984-991, 2021.

[16] B. Bertenyi, "5G Evolution: What's Next?" IEEE Wireless Communications, vol. 28, no. 1, pp. 4-8, 2021.

[17] 3GPP, "RP-211768, Enhancements to Integrated Access and Backhaul for NR," September 2021. [Online]. Available: https://www.3gpp.org/ ftp/tsg_ran/TSG_RAN/TSGR_93e/Docs/RP-211868.zip 